Témoigner Témoigner. Entre histoire et mémoire

Getuigen Revue pluridisciplinaire de la Fondation Auschwitz

$124 \mid 2017$

La musique dans les camps

\title{
Les femmes déportées de France par mesure de répression : nouvelles perspectives
}

De deportatie van vrouwen uit bezet Frankrijk. Nieuwe perspectieven

\section{Pierre-Emmanuel Dufayel}

\section{(2) OpenEdition}

1 Journals

\section{Édition électronique}

URL : https://journals.openedition.org/temoigner/5790

DOI : $10.4000 /$ temoigner.5790

ISSN : 2506-6390

Éditeur:

Éditions du Centre d'études et de documentation Mémoire d'Auschwitz, Éditions Kimé

Édition imprimée

Date de publication : 2 avril 2017

Pagination : 144-154

ISBN : 978-2-930953-00-7

ISSN : 2031-4183

Référence électronique

Pierre-Emmanuel Dufayel, « Les femmes déportées de France par mesure de répression:

nouvelles perspectives », Témoigner. Entre histoire et mémoire [En ligne], 124 | 2017, mis en ligne le 30 novembre 2021, consulté le 01 décembre 2021. URL : http://journals.openedition.org/temoigner/5790 ; DOI : https://doi.org/10.4000/temoigner.5790 


\section{Les femmes déportées de France par mesure de répression: nouvelles perspectives}

$\rightarrow$ Par Pierre-Emmanuel Dufayel Université de Caen BasseNormandie a déportation a représenté pour les femmes vivant en France sous l'Occupation «l'impôt du sang»; la souffrance endurée par des milliers de femmes symbolisant avec force le sacrifice du deuxième sexe pour la défense du pays, et sa participation à la lutte menée par l’armée des ombres. En effet, la déportation rassemble l'immense majorité des femmes victimes des politiques de répression, visées notamment par les mesures de maintien de l'ordre de l'occupant.

Pourtant, jusqu'aux travaux de la Fondation pour la Mémoire de la Déportation réalisés dans le cadre du livre-mémorial dans le milieu des années 1990 qui a permis de mettre à jour les processus de déportation, les seules avancées scientifiques se limitaient encore à étudier le quotidien des déportées dans l'univers concentrationnaire. Durant près d'un demi-siècle, ce sont les déportées elles-mêmes qui ont fourni l'essentiel des travaux. Est-il encore utile de rappeler l'œuvre pionnière de Germaine Tillion qui dès le lendemain de son rapatriement en Suède en avril 1945 entreprend un recensement méthodique des informations ? Un travail qui représente encore aujourd'hui la base incontournable de toutes les recherches sur les déportées de France. Dans le sillage de son maître-livre, Ravensbrück, les problématiques se sont pendant plusieurs décennies focalisées sur le fonctionnement et l'organisation du plus grand camp de concentration pour femmes du Reich dans lequel plus de 100000 prisonnières et plus de 20000 hommes ont été internés, devenu naturellement le lieu de mémoire des femmes réprimées par l'Allemagne nazie. Ainsi, plus de 70 ans après le retour des dernières déportées, ce sont encore les travaux analysant le fonctionnement du camp de Ravensbrück qui dominent la mince bibliographie sur le sujet. Dernièrement, l'immense travail de Bernhard 

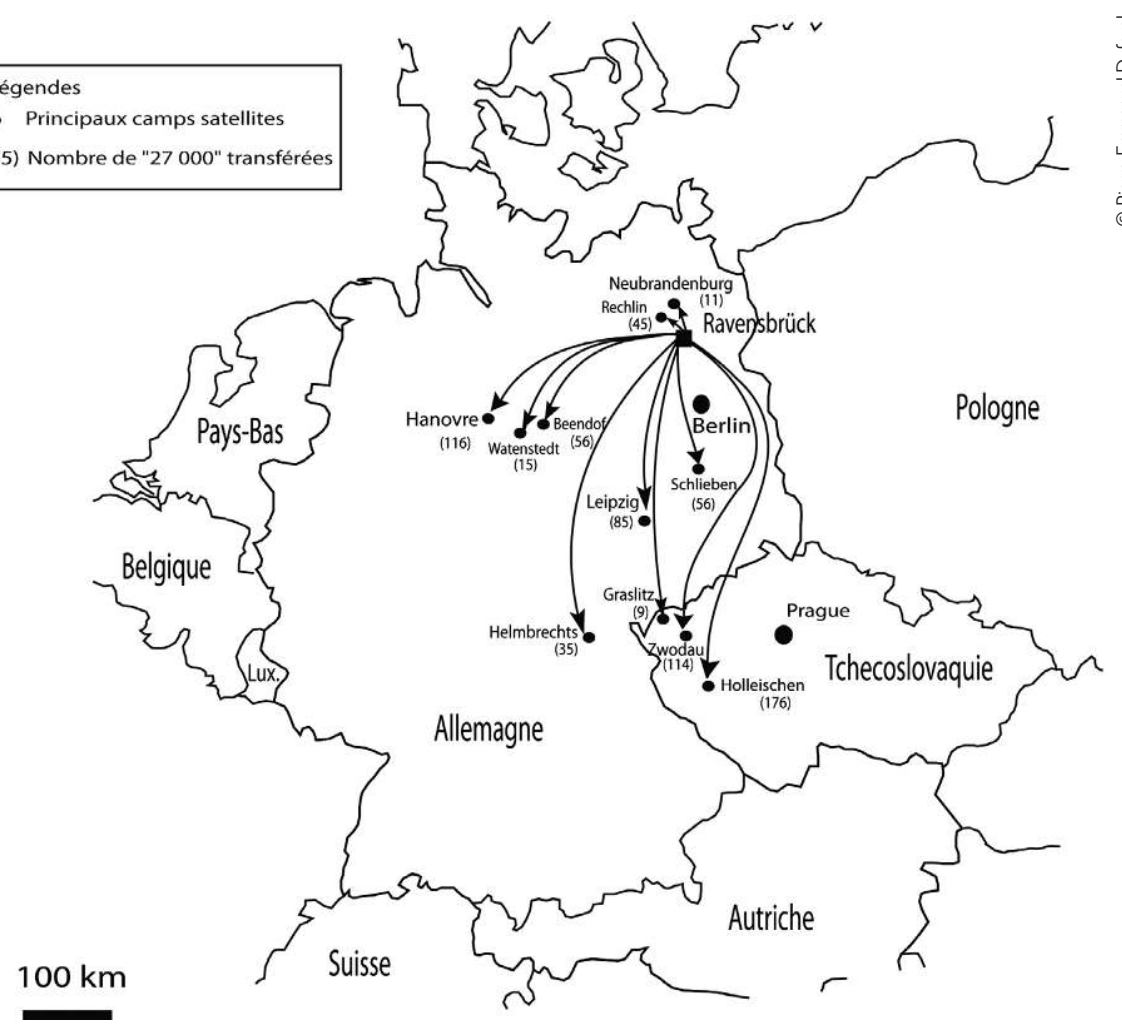

- Dispersion des femmes déportées le 31 janvier 1944 dans le convoi dit des 27000

Strebel (publié en français en juin 2005), intitulé Ravensbrück, un complexe concentrationnaire, nous livre une étude aussi complète que minutieuse sur le camp.

Au cours de ces années, une affirmation a traversé et accompagné l'historiographie : celle d'une histoire sans archives ; affirmation née essentiellement de la destruction des archives du camp de Ravensbrück avant sa libération. L'idée d'une histoire presque impossible ou, pour le moins, difficile à faire à partir de sources écrites en a découlé. Il apparait aujourd'hui évident à la lumière des études récentes que la documentation disponible est bien au contraire très riche, et qu'il est possible d'écrire l'histoire de la déportation des femmes à partir de fonds documentaires largement accessibles. C'est ce que nous tenterons d'expliquer dans notre premier point.

L'ouverture de nouveaux fonds a permis d'ouvrir considérablement le champ de la recherche et de renouveler les problématiques. Dans le sillage de travaux qui éclairent d'un jour nouveau les politiques de répression et de maintien de l'ordre en France occupée comme ceux de Thomas Fontaine ou de Gaël Eismann, la question 


\section{Varia}

_ Danielle Casanova, née Vincentella Perini, Ajaccio, 9 janvier 1909 - Auschwitz, 9 mai 1943. L'une des figures emblématiques du premier convoi de femmes déportées.

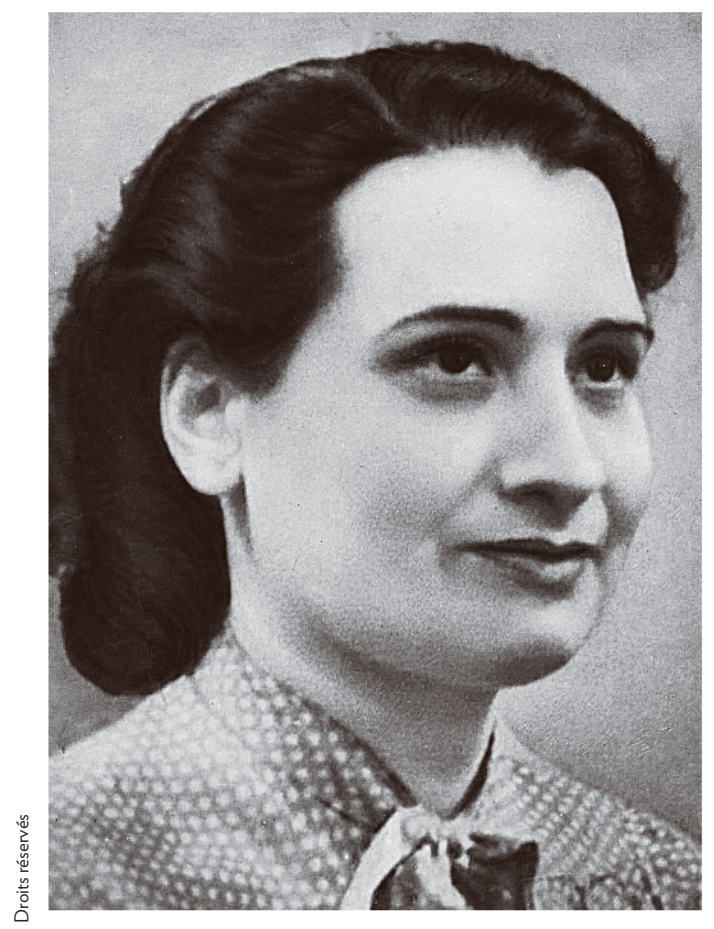

LES FEMMES DÉPORTÉES DE FRANCE PAR MESURE DE RÉPRESSION : NOUVELLES PERSPECTIVES

des processus de déportation des femmes devient essentielle. De même, leur portrait sociopolitique ainsi que leur parcours peuvent être analysés, offrant une définition à la fois plus précise et plus complexe de la déportation.

\section{LA DÉPORTATION DES FEMMES : UNE HISTOIRE SANS ARCHIVES ?}

L'idée selon laquelle l'histoire de la déportation des femmes doit se faire sans archives date de l'immédiat après-guerre. Pour comprendre comment elle s'installe et se cristallise dès cette période charnière, il est primordial de revenir sur le rôle joué par les survivantes dans l'historiographie de la déportation des femmes et en particulier sur celui de Germaine Tillion. Par sa formation d'ethnologue, par son expérience de l'enquête de terrain - Germaine Tillion a passé plusieurs années en Algérie au sein des tribus berbères qu'elle a étudiées -, grâce à son sens aigu de l'observation, elle analyse dès les premiers mois de sa déportation le fonctionnement du camp. Elle cherche à connaitre auprès des détenues employées dans les différents services les chiffres, les effectifs du camp, l'organisation économique. Elle pose un regard sur cette expérience d'une rare lucidité qui lui permet déjà de comprendre et de décrypter des aspects importants du système concentrationnaire. Restée à Ravensbrück jusqu'au bout, Germaine Tillion est témoin comme beaucoup de détenues de la destruction des archives du camp de Ravensbrück. Pendant deux semaines et demie, devant l'imminence de la défaite, les documents de l'administration du camp sont détruits en commençant par la section politique, celle qui détenait sans aucun doute le plus d'informations sur les détenues. Une somme considérable de documents essentiels dans l'écriture de l'histoire de la déportation des femmes est alors perdue. Dès le lendemain de sa libération, Germaine Tillion qui se trouve en Suède interroge les survivantes, rassemble ce qu'elle peut de noms, de matricules et réalise déjà un premier recensement. Pour les déportées qui ont survécu, témoins de la destruction des archives, qui avaient déjà pensé et étudié le fonctionnement du camp, il est crucial dès lors de sauver de l'oubli les noms des victimes. Dès les premières semaines après la Libération, germe dans l'esprit de Germaine Tillion et de ses camarades l'idée qu'il faudra certainement faire l'histoire de la déportation des femmes sans archives. Cette constatation Germaine Tillion la formulera d'ailleurs ainsi quelques années plus tard: «Quand le dernier camp de concentration allemand a ouvert ses portes, cet "autre monde" a cessé de découper sa masse dans l'espace réel pour se profiler parmi les fantômes de la "dimension historique", mais il les rejoignait sans bagages nu comme ses morts.» (Tillion, 1954, p. 38) Elle poursuit 
ainsi après-guerre ses recherches sur cette histoire «sans bagages ». Elle fait d'abord partie de la Commission des déportés et internés politiques et raciaux, elle devient vice-présidente de la Commission d'Histoire de la Déportation à sa création en juin 1951 au sein du comité d'histoire de la Seconde Guerre mondiale. L'histoire est ainsi écrite jusqu’à la fin des années 1970 par les déportées elles-mêmes, essentiellement à partir de leurs témoignages, et de l'enquête orale. Un vaste recueil testimonial réalisé de façon extrêmement méthodique, qui donne des résultats déjà très fiables sur le recensement des victimes et sur la chronologie des transports notamment. Pendant près de 30 ans, Germaine Tillion et plusieurs de ses camarades rassemblent un nombre considérable de témoignages par camps, par convoi, par thèmes et parviennent, autant qu'il est possible de l'imaginer, à combler le préjudice dû à la destruction des archives du camp de Ravensbrück par les souvenirs des survivantes. Durant cette période où les recherches sur la déportation sont les plus vives, où les anciennes déportées sont mobilisées pour écrire leur histoire, l'idée d'une histoire sans archives est aussi entretenue par une méfiance à l'égard du document, notamment allemand. Un sentiment qu'exprime ainsi Germaine Tillion dans la conclusion d'une analyse sur l'apport des témoignages dans l'étude de la déportation : «Le récit le plus passionné, le plus fourmillant d'erreurs, est sans doute encore plus près de la vérité, moins dangereux pour elle en tout cas, que des publications d'archives...» (Tillion, 1954, p. 37) Il est donc légitime de s'interroger sur le mésusage et/ou le non-usage de documentation pourtant à portée de main. Enfin, si cette affirmation est restée aussi présente au cours de ces années c'est en grande partie à cause de la polarisation et de la focalisation des interrogations sur le camp central de Ravensbrück. Or, si une grande majorité des femmes déportées de France passent un jour dans ce camp, l'immense majorité d'entre elles repartent vers d'autres lieux de déportation. Contrairement à la vision d'ensemble qui persiste encore parfois, Ravensbrück n'est pas l'unique destination des déportées. Très peu en réalité y restent pendant toute leur déportation.

Pourtant les sources qui permettent d'écrire l'histoire de la déportation des femmes de France sont nombreuses. D'emblée, il importe de rappeler que des archives du camp de Ravensbrück ont pu nous parvenir. Des listes de transport, des fiches individuelles de détenues, des listes de transferts, des questionnaires d'entrées sont autant de sources inédites qui offrent de nouvelles perspectives. Des recherches récentes ont par ailleurs permis de mettre en lumière le rôle de l'administration militaire et ce faisant de mieux maitriser les archives de ses différents services, notamment celles de la magistrature et des tribunaux militaires. Pour l'étude de la déportation des femmes, on dispose en particulier de plusieurs centaines de dossiers judiciaires. Autres éléments du dispositif répressif, les prisons et les camps d'internement offrent aussi des fonds documentaires considérables. Concernant les femmes notamment, nous disposons des archives du camp de Romainville, principal camp d'internement pour femmes avant le départ pour le système concentrationnaire, rouage essentiel de l'organisation des transports vers Ravensbrück à partir 
de 1943. Le registre dans son intégralité, qui à lui seul permet pour les femmes de reconstituer la majorité des départs en déportation, a pu être conservé. Mentionnons également les fiches individuelles qui nous informent sur l'identité de la détenue et sur les différents transferts. La consultation des fonds constitués par les archives du rapatriement s'avère aussi indispensable. Encore largement sous-exploités, ils rassemblent un grand nombre de documents sur la période qui va de la libération au retour des déportées en France, une période qui peut s'étendre sur plusieurs mois. Ces archives nous renseignent notamment sur le devenir des déportées et nous éclairent en particulier sur les libérations opérées par la Croix-Rouge Internationale et suédoise dans les dernières semaines de la guerre. On y trouve notamment un important fonds photographique, celui des laissez-passer réalisés en Suède. Il faut enfin évoquer les sources produites après-guerre comme les dossiers individuels, constitués par les victimes et leur famille pour obtenir un titre ou une mention ${ }^{1}$. Ces archives aujourd'hui très largement communicables et accessibles permettent un renouvellement des questionnements tant sur les déportées que sur les déportations.

\section{DES DÉPORTATIONS DE FEMMES}

Entre 1940 et 1944, environ 6700 femmes sont déportées depuis les territoires occupés, et près de 2200 depuis les territoires de l'Alsace et de la Moselle annexés. Nous traiterons essentiellement dans notre développement de la déportation depuis les zones occupées. La répression et les déportations opérées depuis l’Alsace-Moselle annexée, répondant à des logiques propres, font l'objet de travaux spécifiques en particulier ceux de Cédric Neveu. Les politiques répressives au cours de l'Occupation évoluent. Loin d'adopter un schéma simple et uniforme tout au long de la période, cette déportation revêt en réalité de nombreux visages. Les déportations de femmes s'opèrent d'abord dans le cadre d'une répression judiciaire menée par l'administration militaire allemande mise en place dans les territoires occupés [le MBF (Militärbefehlshaber in Frankreich; Commandant militaire allemand en France) ou le MBB (Militärbefehlshaber in Belgien und Nord Frankreich; Commandant militaire allemand en Belgique et dans le nord de la France)]. Les premières déportées de France sont ainsi des femmes condamnées par les tribunaux militaires allemands et conduites dans les prisons et les forteresses du Reich pour y purger leur peine. Françoise de Hauteclocque, l'une des premières femmes déportées de France, est par exemple arrêtée le 4 août 1940 à Ste-Suzanne-par-Prétôt dans la Manche, par la Feldgendarmerie, pour détention d'armes et refus d'obtempérer. Jugée le lendemain par un conseil de guerre de la $12^{\mathrm{e}}$ division d'infanterie, elle est condamnée à 6 mois de prison et déportée dès le 6 août à la prison de Cologne où elle purge sa peine avant d'être libérée.

(1) Conservées à Caen à la Division des Archives des Victimes des Conflits Contemporains, Service Historique de la Défense (DAVCC - SHD).
Dès 1941, les condamnations se font de plus en plus sévères face à une résistance qui progressivement se structure. Dans l'ensemble des zones occupées, plus de 150 condamnations sont prononcées à l'encontre de femmes qui seront ensuite 


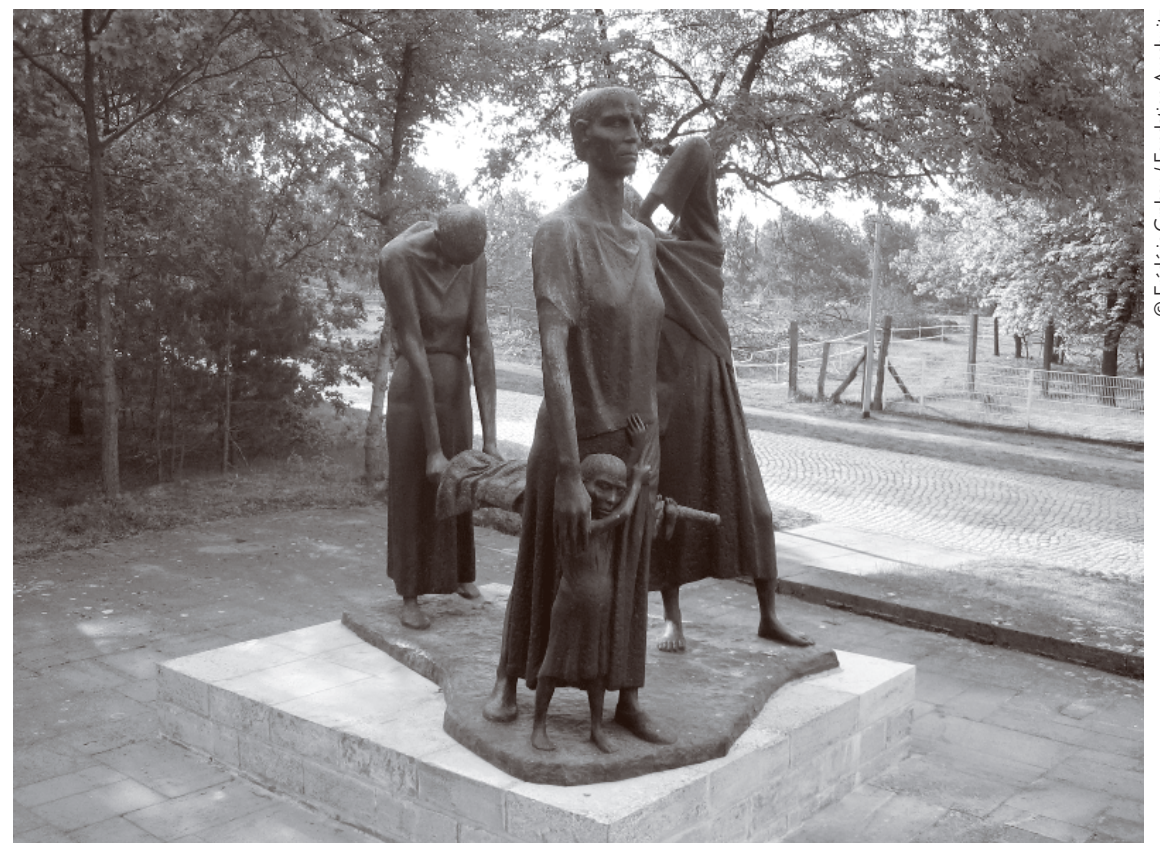

déportées ; au moins 110 d'entre elles sont transférées en Allemagne dès 1941. Des femmes sont aussi pour la première fois condamnées à la peine capitale. À titre d'exemple, Kate Bonnefous, arrêtée le 13 novembre 1940 pour avoir aidé des prisonniers de guerre évadés, est condamnée à mort le 7 mars 1941 par le tribunal militaire du Grand Paris. Déportée le 7 juillet à Trèves, elle est ensuite internée pendant près de trois ans dans les prisons allemandes. Comme pour Kate Bonnefous, la peine de mort sera dans la majorité des cas commuée en réclusion à perpétuité, la procédure interdisant de fusiller en France les femmes condamnées à la peine capitale. Toutefois certaines résistantes membres notamment d'organisations communistes et de groupe de francs-tireurs ne sont pas épargnées. Françoise BlochSerazin, par exemple, agissant au sein du groupe FTP parisien dès les premiers mois de la guerre est arrêtée le 15 mai 1942 dans une souricière installée dans son propre appartement par la brigade spéciale de la police française. Condamnée à mort par le tribunal militaire allemand du Grand Paris le 30 septembre 1942, elle est déportée le 16 novembre 1942 vers Lübeck puis Hambourg où sa peine est finalement mise à exécution le 12 février 1943. Olga Bancic, émigrée roumaine juive et résistante du groupe de Missak Manouchian est aussi transférée en Allemagne après avoir été condamnée à mort comme ses camarades de l'Affiche rouge. Elle est décapitée à Stuttgart le 10 mai 1944. Tout comme Syma Schloss ouvrière d'origine polonaise, guillotinée à Cologne après avoir été condamnée à mort le 24 avril 1942 à Paris pour l'impression et la distribution de tracts dans la résistance communiste.
_ Ravensbrück. Sculpture Tragende de Will Lammert (1959). 
police allemande pour transférer avec le secret offert la procédure des détenues vers les camps de concentration via une prison.

Au mois de juin 1942, laSipo-SD qui prend alors en charge les affaires policières à la place de l'administration militaire en France instaure un nouveau dispositif répressif dont la pierre angulaire devient peu à peu la déportation vers les camps de concentration. Au début de l'année 1943, elle s'accorde officiellement le droit d'interner sans jugement les suspects grâce au principe de la Schutzhaft (détention de sécurité). Dès lors, après plusieurs mois passés dans les prisons de France, l’immense majorité des femmes déportées sont rassemblées à Compiègne puis dans le fort de Romainville qui devient à partir de 1944 l'antichambre de la déportation pour les femmes de France. Le premier convoi direct de femmes est ainsi formé le 23 janvier 1943. Dirigé vers Auschwitz, il emporte 230 femmes dont une grande majorité d'illustres militantes communistes parmi lesquelles Danielle Casanova, Charlotte Delbo, Marie-Claude Vaillant-Couturier. Au cours de cette même année, deux autres transports sont dirigés cette fois vers le camp de Ravensbrück. En 1944, environ 4800 femmes sont déportées depuis les territoires occupés soit les deux tiers des femmes déportées de France dans le cadre des politiques de répression. Le 31 janvier, le plus important départ de femmes sous l'Occupation est formé avec 959 femmes. Au cours du printemps 1944, les Allemands organisent des départs de 50 à 60 prisonnières vers le camp de la Neue Bremm à Sarrebruck où des transports plus importants sont formés à destination cette fois de Ravensbrück. Néanmoins, la déportation des femmes condamnées par des tribunaux militaires se poursuit. Plus d'une centaine sont ainsi transférées depuis la France vers des prisons allemandes. À l'automne 1944, après l'abrogation du décret «NN», l’immense majorité des prisonnières encore présentes dans les geôles allemandes sont transférées vers Ravensbrück.

\section{QUI SONT LES FEMMES DÉPORTÉES DE FRANCE ?}

La connaissance du profil sociopolitique des femmes déportées révèle en réalité un double enjeu : le premier est d'abord de savoir qui tombe sous les coups de la procédure et de connaître ceux que les Allemands décident de déporter, le second est de redonner une identité aux femmes victimes de ces politiques de répression. L'ouverture des dossiers de demande de statut de déporté politique et résistant, associée à la documentation conservée par la Division des Archives des Victimes des Conflits Contemporains (DAVCC) sur les femmes n'ayant pas constitué de dossier après-guerre, a permis de retrouver les motifs d'arrestation, et les activités menées avant celle-ci, de 5832 femmes sur les 6672 déportées de France depuis les territoires occupés, soit $88 \%$.

Si les déportées de France, ont à l'évidence, en grande majorité combattu l'occupant et/ou le régime de Vichy, les sources étudiées permettent aujourd'hui d'affiner considérablement nos connaissances et de redonner du sens au terme de « résis- 
tantes ». En premier lieu, on relève qu'environ $64 \%$ des déportées sont membres d'une organisation de résistance ${ }^{3}$. Près de la moitié sont membre de réseaux, $20 \%$ agissaient au sein d'un mouvement, et environ $30 \%$ étaient membres d'une organisation liée à la résistance communiste (FTPF, Front National par exemple). L'analyse exhaustive des agissements et du rôle des femmes au sein des organisations révèle la diversité des actions menées, mais aussi leurs spécialités. Ainsi en dehors des missions liées au service social (2\%), aux réceptions des parachutages $(2 \%)$ ou à la préparation des actes de sabotage (3\%), les femmes déportées œuvrent également à l'exfiltration des prisonniers en fuite, des aviateurs tombés en territoire ennemi, ou des agents devant rejoindre l'Angleterre (7\%). Les tâches associées au renseignement, du détournement de documents officiels à l'observation des déplacements des troupes allemandes en passant par l'interception du courrier adressé à la Kommandantur, relient près de $15 \%$ des déportées engagées dans une organisation de résistance, alors que plus d'une femme sur cinq a pris part à des actions de propagande (distribution, impression, rédaction). Cependant, l'activité de ces résistantes semble surtout s'articuler autour de deux spécialités. En premier lieu, elles sont environ $44 \%$ à avoir servi d'agent de liaison pour la transmission et le transport des messages, des armes, des documents, et du matériel en tous genres. Enfin, plus d'une femme sur deux a assuré l'hébergement des résistants traqués ou des personnes recherchées. Dans sa forme la plus complète, ce rôle consistant fondamentalement à offrir la protection et la sécurité du foyer familial, soit à assumer en somme les tâches quotidiennes, s'accompagne de surcroît, dans bien des cas, du camouflage des armes, des documents ou du matériel, de la garantie d'un lieu de rassemblement pour les réunions, ou de l'hébergement d'émissions radio. Qu’elle se nomme «résistance au foyer», ou intendance, cette forme de lutte demeure au cœur même de la spécificité du combat féminin, découlant elle-même du rôle et de la fonction attribués aux femmes dans la société des années 1940.

Par ailleurs, aux côtés de celles qui ont intégréles rangs de la Résistance organisée se trouvent les femmes qui composent cette résistance qu'il est convenu d'appeler la «résistance civile», d'après l'expression propre à l'historien Jacques Sémelin. Se trouve ici représentée la partie de l'opinion rejetant l'Occupation et qui soutient dans l'ombre la Résistance sans en faire partie. Par son caractère spontané et non structuré, cette composante demeure généralement difficile à faire émerger. L'étude de la répression permet ici de révéler ces comportements. Ainsi $43 \%$ de ces femmes ont soutenu la Résistance, en logeant spontanément des agents de passage, en camouflant pour quelques jours des résistants recherchés ou en cachant armes et matériel. Au moins $37 \%$ parmi elles viennent en aide aux pilotes, aux soldats alliés, ou aux personnes traquées, alors que $20 \%$ des femmes de la résistance civile ont été arrêtées pour la simple expression de sentiments anti-allemands ou pro-alliés.

(3) Les statistiques présentées ont été établies grâce à l'ouverture des dossiers statut, DAVCC - SHD.
Les résistantes prises dans leur globalité de sensibilités, de gestes et d'actions représentent ainsi environ $87 \%$ des déportées. Toutefois, cette prépondérance ne 
doit pas occulter une part non négligeable de femmes qui ne sont pas arrêtées pour des actes de résistance. Dans ce groupe minoritaire, un tiers est interpellé pour des délits de droit commun. Si les habituelles affaires de vols et de marché noir provoquent l'arrestation d'un certain nombre d'entre elles, la très grande majorité sont des femmes arrêtées dans le cadre des mesures prises pour contrôler les relations sexuelles entre les Françaises et les troupes d'occupation et garantir la sécurité sanitaire du territoire occupé. Le reste des femmes n'étant pas arrêtées pour des actes de résistance sont pour la plupart des mères, des sœurs, ou des proches de résistants traqués très certainement victimes de la procédure de la Sippenhaft : procédure par laquelle l'entourage du résistant est tenu responsable tout autant que le résistant lui-même.

Par ailleurs, les informations recueillies permettent une approche sociologique relativement détaillée de ces déportées. L'étude des origines géographiques révèle leur diversité. Cependant la prépondérance des citadines apparait clairement : les habitantes des villes de Paris, Marseille et Lyon représentent à elles seules près d'un quart des déportées. L'analyse des classes d'âge montre d'autre part que les déportées étaient dans la force de l'âge : les femmes de 30 à 45 ans sont les plus surreprésentées par rapport à la population française, la moyenne d'âge s'élève à 37 ans. Une réalité qui semble donc bien éloignée de l'image de la très jeune combattante de l'ombre composant encore très largement les représentations de la résistante. L'examen de la situation familiale comme celui des catégories socioprofessionnelles vient également contrarier cette vision. En effet, environ $55 \%$ des déportées sont mariées au moment de leur arrestation, alors que seulement un tiers d'entre elles se déclarent célibataires. L'étude de la répartition par professions enfin démontre qu'elles sont dans la grande majorité des cas des femmes actives (66\%), ce qui les distingue là encore de la population féminine française dans laquelle plus d'une femme sur deux est alors sans profession.

\section{PARCOURS ET BILAN D'UNE RÉPRESSION}

La question du parcours des femmes est centrale puisqu'elle fait émerger, d'une part, les logiques économiques de cette déportation et démontre, d'autre part, la nécessité de décentrer notre regard du camp central de Ravensbrück.

L'étude du parcours de ces déportées révèle en effet une géographie de la déportation aux lieux multiples, qu'il s'agisse bien sûr des nombreux camps satellites, ou Kommando de travail, mais également des prisons et des forteresses dans lesquelles les femmes de France sont internées. Jeanne Bonneaux, arrêtée le 15 mars 1942 à Paris pour son activité dans la Résistance, est par exemple transférée dans huit prisons différentes avant d'entrer le 21 novembre 1944 à Ravensbrück. Certaines déportées comme Marie-Louise Le Bozec connaitront jusqu’à 12 lieux de déportation. Près de 1900 femmes passent par une prison pendant leur parcours alors qu'au 
(4) SHD - DAVCC. D’après le dépouillement des dossiers de demande de statut.

\section{BIBLIOGRAPHIE}

- Dufayel, Pierre-Emmanuel (2011): Un convoi de femmes, Paris, Vendémiaire.

- Eismann, Gaël (2010) : Hôtel Majestic. Ordre et sécurité en France occupée (1940-1944), Paris, Tallandier.

- Fondation pour la Mémoire de la Déportation (2004) : Livre-Mémorial des déportés de France arrêtés par mesure de répression et dans certains cas par mesure de persécution. 1940-1945, Paris, Tirésias.

- Fontaine, Thomas (2005) : Les oubliés de Romainville, Paris, Tallandier.

- Lacour-Astol, Catherine (2015) : Le genre de la Résistance, Paris, Presses de la Fondation nationale de sciences politiques.

- Strebel, Bernhard (2005) : Ravensbrück, un complexe concentrationnaire, Paris, Fayard, traduit de l'allemand par Odile Demange.

- Tillion, Germaine (1988) : Ravensbrück [1973], Paris, Seuil.

- Tillion, Germaine (juilletseptembre 1954) : "Réflexion sur l'étude de la déportation », in Revue d'Histoire de la Seconde Guerre mondiale, $4^{\mathrm{e}}$ année, $\mathrm{n}^{\circ} 15$, p. 3-38. moins 900 d'entre elles n'entrent pas dans un camp de concentration ${ }^{4}$. L'étude de ces centaines de parcours illustre nettement la dimension plurielle de la déportation et l'éclatement des expériences vécues. Les prisons de Jauer, Cottbus, Gotteszel, Aichach, Flüssbach sont autant de lieux qu'il est indispensable d'appréhender et de connaitre pour mieux définir les contours de cette répression.

Par ailleurs, Ravensbrück devient au cours de l'année 1943 une immense plaque tournante de la répartition des femmes de toute l'Europe dans l'industrie de guerre allemande. Plus des deux tiers des déportées de France qui entrent dans le camp sont transférés dans une usine d'armement, dans une zone qui s'étend de la Baltique aux Sudètes. La répartition des femmes du transport parti le 31 janvier de Compiègne et qui arrive le 2 février à Ravensbrück est parfaitement caractéristique de cette dispersion des prisonnières. Parmi elles, plus de sept femmes sur dix sont transférées dans un Kommando de travail. Dès le mois d'avril, environ 300 femmes du convoi sont d'abord envoyées dans les Sudètes à Holleischen et à Zwodau, au mois de juillet plus d'une centaine partent pour le camp Limer à Hanovre, alors que quinze jours plus tard presque autant quittent Ravensbrück pour Leipzig. En définitive, on estime que plus de 70 lieux de déportation sont liés à la déportation des femmes de France. L'analyse du parcours fait d'autre part ressortir des parcours individuels singuliers comme celui des femmes enceintes dont une partie pourra être rapatriée en France pour accoucher à l'été 1944 .

L'étude du devenir des déportées permet enfin d'établir un bilan précis de cette répression. Dans l'état actuel des recherches, on comptabilise 1533 femmes disparues ou décédées en déportation, soit $24 \%$ des femmes déportées depuis la France occupée. Le sort de 184 femmes demeure encore à ce jour inconnu. Plus de la moitié de ces femmes décèdent entre le $1^{\text {er }}$ février et le 30 avril 1945, au cours d'une période où l'extermination des plus faibles est organisée dans le camp central de Ravensbrück. Dans six cas sur dix, Ravensbrück est le lieu de décès des femmes déportées.

Ainsi, il apparait que ce renouvellement des problématiques sur la déportation des femmes permet de recomposer une histoire fragmentée en remettant dans une même perspective résistance, politique répressive et système concentrationnaire. Mais il offre également une vision à la fois plus précise et plus complète, nous éloignant un peu plus d'un schéma d'ensemble uniforme et unisexe qui écrase les différences, éclairant les spécificités de la déportation des femmes qui reste encore aujourd'hui l'une des zones d'ombre de l'histoire des femmes et du genre. 